\title{
A Preliminary Study on Birdlife of Betana Wetland, Belbari, Morang District, SE-Nepal
}

\author{
Milan Kharel* \\ Department of Zoology, Central Campus of Technology, Tribhuvan University, Dharan.
}

\begin{abstract}
Received: 27 September, 2018; Accepted: 12 November, 2018; Published: 15 November, 2018
*Corresponding author: Milan Kharel, Department ofZoology, Central Campus of Technology, Tribhuvan University, Dharan, E-mail: milankharel2000@ gmail.com
\end{abstract}

\begin{abstract}
The present study conducted in Betana wetland, Morang, Nepal. The study based on the field survey in order to find out the avifaunal composition. Most of the birds were found belong to the order Passeriformes including nine families and 15 species, followed by Coraciformes with three families and four species then Pelicaniformes with two families and six species. Of them, two species viz. Greyheaded fish eagle (Icthyophaga humilis) and Lesser Adjutant Stork (Leptoptilos javanicus) are kept under Near Threatened (NT) and Vulnerable (VU) category of IUCN Red List of threatened species. About $70 \%$ of total bird recorded was resident type and about 35\% of total recorded bird species were fairly common in abundance. The species richness and abundance of avifauna found higher in winter season than summer. Compared to the previous study, birds belonging to four more families and five more orders recorded this time.
\end{abstract}

Keywords: Betana; avifauna; wetland; Icthyophaga humilis; Leptoptilos javanicus; Terai

\section{Introduction}

After forest, more than a quarter (27\%) of Nepal's nationally threatened birds inhabits in wetlands. Out of 886 species of Bird species recorded so far in Nepal, about 42 species recorded in Nepal are listed in IUCN Red List of globally threatened birds, 35 globally near threatened and 167 species are nationally threatened [2]. More than 230 species of birds have been found to be wetland-dependent in Nepal [10]. Wetlands are among the most productive ecosystem in the world. The wetlands of Nepal well known for their unusually rich biodiversity. They occupy approximately five percent of the total area of Nepal in the form of rivers, stream, lakes, reservoirs, village ponds, paddy fields, marsh and swampland. There are over 405 wetland areas in Nepal from the Terai to the Himalayas. The loss of diversity of the water birds reduces the natural resource base of the country. Wetlands are one of the most threatened habitats because of their vulnerability and attractiveness for development [7]. Betana wetland is an important recreational destination with great potential of wildlife including bird diversity. Now this area has been established as a picnic spot and other recreational activities that have created lots of problem on habitat, breeding and feeding activities of birds. Firewood collection and grazing are the prominent human-induced disturbances of this area. The forest area of wetland dominated by Sal (Shorea robusta) followed by Khair-Sissoo (Acacia catechu-Dalbergia sissoo) and mixed forest. Grassland flora consisted of a combination of wetland herbs and moist grass species which are commonly composed of Imperata cylindrica, Cyperus papyrus, Digitariya ciliaris, Bulbostylis barabata, Erasgrostis tentella, Cyperus totundus, Polygonum spp. etc. [11]. Data on the avifaunal composition of this area is not adequate to assess conservation needs. Only a little information from records of bird watchers, nature guides etc. are available [11]. Hence, the present study was essential to provide baseline data on avifaunal composition for the proper conservation and management initiatives.

\section{Study Area}

Betana wetland is a freshwater pond situated between $26.659106^{\circ} \mathrm{N}$ to $87.428814^{\circ} \mathrm{E}$ and $26.662894^{\circ} \mathrm{N}$ to $87.434018^{\circ}$ $\mathrm{E}$ at an elevation of $123 \mathrm{~m} \mathrm{msl}$, covering 5.5 ha area in Belbari municipality of Morang district. It is one kilometer far from the Belbari Bazar in the east. The wetland area remains surrounded by Sal forest from east, north and west sides whereas Mahendra highway lies adjacent on its south. The depth of the pond varies from 0.5 to $1.5 \mathrm{~m}$ in the dry season and 1 to $2.5 \mathrm{~m}$ in monsoon season [9]. The study area experiences a tropical monsoon climate with winter, summer and rainy seasons in a year. The soil is alluvial type and the average annual temperature of 24.6 ${ }^{\circ} \mathrm{C}$. The average rainfall is $2256 \mathrm{~mm}$ per year and about $90 \%$ of rainfall occurs within three months of monsoon seasons (JuneAugust).

\section{Materials and Methods}

Study of the avifaunal composition of Betana wetland carried out for six months from February 2017 to July 2017. Therefore, the birds of summer, winter and monsoon season could be observed within a short field visit period. Regular visits of study site done almost once a week on every Saturday morning between $7-10$ am and 4-6 pm in the evening in order to know the avifaunal composition. The bird census was done by applying the Point Count Survey Method within the radius of 50m [6]. In the direct count method, counts performed in the four sampling stations (F1, F2, W1 and W2) repeatedly. Some birds were photographed with the help of Canon Power shot SX520 HS 42x 
24-1008mm 16 MP optical zoom digital camera. The primary data were collected by direct observation of species with the help of Bushnell $\mathrm{H}_{2} \mathrm{O}$ Waterproof/Fogproof Prism Binocular 10 x42 $\mathrm{mm}$. While secondary data collected by the help of questionnaire, reviewing literatures such as journals, articles, proceedings and books. The geographical coordinates were taken by using Garmin eTrex 10 Worldwide Handheld GPS navigator. The identification of birds was done by direct observation method. Birds were observed within transect of $100 \mathrm{~m}$ and identified with the help of field guide books of Ali, et al. 1986, Fleming, et al. 2000, Shrestha, et al. 2000, Grimmett, et al.2000, Grimmett, et al. 2016, Pradhan. et al.2018 [1,3,4,5,8,10]. Photograph of unidentified species were identified with the help of subject expertise of Post-graduate campus, Biratnagar. For the birds, which are shy and could not be observed directly, the call count method was employed for their identification. The study area was divided into four pockets (Map1). Bird observation was done at these four pockets viz. the forest area F1 (west) and forest area F2 (east), wetland area W1 (east) and wetland area W2 (west) of Betana wetland area. Four line transects were set along the two forests sampling areas (F1 and F2) and two wetland areas (W1 and W2). Each pocket was used as the reference points for the point count method. Observations of birds were carried out on each $50 \mathrm{~m}$ radius of each pocket.

\section{Result and Discussion}

In the present study, a total of 49 species of birds belonging to 30 families and 15 orders have been reported from Betana wetland based on field observation (Table 1). Among them, 34 species $(69.38 \%)$ of birds were of the resident type, 9 species $(18.36 \%)$ were winter visitor and 6 species (12.24\%) were summer visitors (Figure 3 ). Of them, $34.69 \%$ species $(n=17)$ were fairly common, $24.48 \%$ species $(n=12)$ of birds were common, $22.44 \%(n=11)$ species were occasional and $18.36 \%$ $(\mathrm{n}=9)$ species were uncommon (Figure 4).

\begin{tabular}{|c|c|c|c|c|c|c|c|}
\hline S.No & Order & Family & Common name & Scientific name & Local name & SS & $\mathbf{A B}$ \\
\hline 1 & Accipitriformes & Accipitridae & Black kite & $\begin{array}{l}\text { Milvus migrans } \\
\text { (Boddaert,1783) }\end{array}$ & Kalocheel & WV & UC \\
\hline 2 & Accipitriformes & Accipitridae & $\begin{array}{l}\text { Grey-headed Fish } \\
\text { eagle }\end{array}$ & $\begin{array}{l}\text { Icthyophaga humilis a } \\
\text { (Muller,S \& Schlegel, } \\
\text { 1841) }\end{array}$ & Machha-kul & $\mathrm{R}$ & 0 \\
\hline 3 & Accipitriformes & Pandionidae & Osprey & $\begin{array}{l}\text { Pandion haliaetus } \\
\text { (Linnaeus,1758) }\end{array}$ & Malaha cheel & WV & UC \\
\hline 4 & Accipitriformes & Accipitridae & $\begin{array}{l}\text { Crested Serpent } \\
\text { Eagle }\end{array}$ & $\begin{array}{l}\text { Spilornis cheela } \\
\text { (Latham,1790) }\end{array}$ & Kakakul & $\mathrm{R}$ & UC \\
\hline 5 & Anseriformes & Anatidae & Common Teal & $\begin{array}{c}\text { Anas crecca } \\
\text { (Linnaeus,1758) }\end{array}$ & Vijula Gairi & WV & $\mathrm{C}$ \\
\hline 6 & Anseriformes & Dendroygnidae & $\begin{array}{l}\text { Lesser Whistling } \\
\text { Duck }\end{array}$ & $\begin{array}{l}\text { Dendrocygna javanica } \\
\text { (Horsfield, 1821) }\end{array}$ & Silsile & WV & UC \\
\hline 7 & Bucerotiformes & Upupidae & Common Hoopoe & $\begin{array}{c}\text { Upupa epops } \\
\text { (Linnaeus,1758) }\end{array}$ & Fafre chara & $\mathrm{R}$ & $\mathrm{FC}$ \\
\hline 8 & Charadriiformes & Charadriidae & $\begin{array}{l}\text { Red-wattled } \\
\text { Lapwig }\end{array}$ & $\begin{array}{l}\text { Vanellusindicus } \\
\text { (Boddaert,1783) }\end{array}$ & Huttityaun & $\mathrm{R}$ & $\mathrm{C}$ \\
\hline 9 & Charadriiformes & Jacanidae & $\begin{array}{l}\text { Bronze-winged } \\
\text { Jacana }\end{array}$ & $\begin{array}{l}\text { Metopidiusindicus } \\
\text { (Latham, 1790) }\end{array}$ & Lama aunle & $\mathrm{R}$ & FC \\
\hline 10 & Ciconiiformes & Ciconiidae & Lesser Adjutant & $\begin{array}{l}\text { Leptoptilos javanicus b } \\
\text { (Horsfield,1821) }\end{array}$ & Bhundifor garud & $\mathrm{R}$ & 0 \\
\hline 11 & Ciconiiformes & Ciconiidae & Asian Open bill & $\begin{array}{l}\text { Anastomus oscitans } \\
\text { (Boddaert,1783) }\end{array}$ & Ghungifor garud & SV & UC \\
\hline 12 & Columbiformes & Columbidae & Rock Pigeon & $\begin{array}{l}\text { Columba livia } \\
\text { (Gmelin, } 1789\end{array}$ & Parewa & $\mathrm{R}$ & $\mathrm{FC}$ \\
\hline 13 & Columbiformes & Columbidae & Spotted dove & $\begin{array}{l}\text { Streptopelia chinensis } \\
\text { (Scopoli, 1786) }\end{array}$ & Kurle dhukur & $\mathrm{R}$ & $\mathrm{FC}$ \\
\hline 14 & Columbiformes & Columbidae & $\begin{array}{c}\text { Eurasian Collared } \\
\text { Dove }\end{array}$ & $\begin{array}{l}\text { Streptopelia decaocto } \\
\text { (Frivaldszky, 1838) }\end{array}$ & Kanthe dhukur & $\mathrm{R}$ & $\mathrm{FC}$ \\
\hline 15 & Coraciiformes & Alcedinidae & $\begin{array}{l}\text { Stork-billed } \\
\text { Kingfisher }\end{array}$ & $\begin{array}{l}\text { Halcyon capinsis } \\
\text { (Linnaeus,1766) }\end{array}$ & Thulomatikore & $\mathrm{R}$ & 0 \\
\hline 16 & Coraciiformes & Coraciidae & Dollar bird & $\begin{array}{l}\text { Eurystomus orientalis } \\
\text { (Linnaeus, 1766) }\end{array}$ & Lal chuche theuwa & SV & 0 \\
\hline
\end{tabular}




\begin{tabular}{|c|c|c|c|c|c|c|c|}
\hline 17 & Coraciiformes & Alcedinidae & $\begin{array}{l}\text { White-throated } \\
\text { Kingfisher }\end{array}$ & $\begin{array}{l}\text { Halcyon smyrnensis } \\
\text { (Linnaeus, 1758) }\end{array}$ & Setokanthematikore & $\mathrm{R}$ & 0 \\
\hline 18 & Coraciiformes & Meropidae & $\begin{array}{l}\text { Chestnut-headed } \\
\text { Bee-eater }\end{array}$ & $\begin{array}{l}\text { Merops leschenaultia } \\
\text { (Linnaeus,1758) }\end{array}$ & $\begin{array}{l}\text { Katus tauke Murali } \\
\text { chara }\end{array}$ & SV & UC \\
\hline 19 & Cuculiformes & Cuculidae & Indian Cuckoo & $\begin{array}{l}\text { Cuculus micropterus } \\
\text { (Gould, 1838) }\end{array}$ & Kaphal pakyo & SV & 0 \\
\hline 20 & Cuculiformes & Cuculidae & Greater Couckal & $\begin{array}{l}\text { Centropus sinensis } \\
\text { (Stephens, 1815) }\end{array}$ & Dhade gokul & WV & $\mathrm{C}$ \\
\hline 21 & Gruiformes & Rallidae & $\begin{array}{l}\text { White-brested } \\
\text { Waterhen }\end{array}$ & $\begin{array}{l}\text { Amaurornis phoenicurus } \\
\text { (Pennant, 1769) }\end{array}$ & Sim kukhura & $\mathrm{R}$ & $\mathrm{FC}$ \\
\hline 22 & Passeriformes & Corvidae & Large-billed Crow & $\begin{array}{l}\text { Corvus macrorhynchos } \\
\text { (Wagler,1827) }\end{array}$ & Kalokag & $\mathrm{R}$ & $\mathrm{C}$ \\
\hline 23 & Passeriformes & Corvidae & Rufous Treepie & $\begin{array}{l}\text { Dendrocitta vagabunda } \\
\text { (Latham,1790) }\end{array}$ & Kokale & $\mathrm{R}$ & 0 \\
\hline 24 & Passeriformes & Corvidae & House Crow & $\begin{array}{l}\text { Corvus splendens } \\
\text { (Vieillot, 1817) }\end{array}$ & Gharkag & $\mathrm{R}$ & $\mathrm{FC}$ \\
\hline 25 & Passeriformes & Dicuridae & Black Drongo & $\begin{array}{l}\text { Dicrurus macrocercus } \\
\text { (Vieillot, 1817) }\end{array}$ & Kalochibe & $\mathrm{R}$ & FC \\
\hline 26 & Passeriformes & Dicuridae & Crow billed Drongo & $\begin{array}{l}\text { Dicrurus annectans } \\
\text { (Hodgson,1838) }\end{array}$ & Kagthude chibe & SV & FC \\
\hline 27 & Passeriformes & Dicuridae & $\begin{array}{l}\text { Geater Racket- } \\
\text { tailed Drongo }\end{array}$ & $\begin{array}{l}\text { Dicrurus paradiseus } \\
\text { (Linnaeus, 1766) }\end{array}$ & Bhimraj chibe & $\mathrm{R}$ & $\mathrm{C}$ \\
\hline 28 & Passeriformes & Oriolidae & Golden Oriole & $\begin{array}{l}\text { Oriolus oriolus } \\
\text { (Linnaeus,1758) }\end{array}$ & Gajale sunchari & SV & UC \\
\hline 29 & Passeriformes & Oriolidae & $\begin{array}{l}\text { Black- headed } \\
\text { Oriole }\end{array}$ & $\begin{array}{l}\text { Oriolus xanthornus } \\
\text { (Linnaeus, 1758) }\end{array}$ & Kalotauke sunchari & $\mathrm{R}$ & UC \\
\hline 30 & Passeriformes & Sturnidae & Common Myna & $\begin{array}{l}\text { Acridotheres tristis } \\
\text { (Linnaeus, 1766) }\end{array}$ & Dangrerupi & $\mathrm{R}$ & $\mathrm{FC}$ \\
\hline 31 & Passeriformes & Sturnidae & Asian Pied Starling & $\begin{array}{l}\text { Gracupica contra } \\
\text { (Linnaeus, 1758) }\end{array}$ & Danger saraun & $\mathrm{R}$ & $\mathrm{FC}$ \\
\hline 32 & Passeriformes & Laniidae & Long-tailed Shrike & $\begin{array}{l}\text { Lanius schach } \\
\text { (Linnaeus, 1758) }\end{array}$ & Bhadrai & WV & UC \\
\hline 33 & Passeriformes & Passeridae & House Sparrow & $\begin{array}{l}\text { Passer domesticus } \\
\text { (Linnaeus,1758) }\end{array}$ & Bhagera & $\mathrm{R}$ & $\mathrm{FC}$ \\
\hline 34 & Passeriformes & Pycnonotidae & Red-vented Bulbul & $\begin{array}{l}\text { Pycnonotus cafer } \\
\text { (Linnaeus, 1766) }\end{array}$ & Jureli & $\mathrm{R}$ & FC \\
\hline 35 & Passeriformes & Muscicapidae & $\begin{array}{l}\text { Oriental Magpie } \\
\text { Robin }\end{array}$ & $\begin{array}{l}\text { Copsychus saularis } \\
\text { (Linnaeus, 1758) }\end{array}$ & Dhobi chara & $\mathrm{R}$ & $\mathrm{FC}$ \\
\hline 36 & Passeriformes & Leiothrichidae & Jungle Babbler & $\begin{array}{l}\text { Turdoides striata } \\
\text { (Dumont, 1823) }\end{array}$ & Bagale vyakur & $\mathrm{R}$ & $\mathrm{C}$ \\
\hline 37 & Pelicaniformes & Ardeidae & Indian Pond Heron & $\begin{array}{c}\text { Ardeola grayii } \\
\text { (Sykes,1832) }\end{array}$ & Askote bakulla & $\mathrm{R}$ & FC \\
\hline 38 & Pelicaniformes & Ardeidae & Purple Heron & $\begin{array}{l}\text { Ardea purpurea } \\
\text { (Linnaeus,1766) }\end{array}$ & Dhyani bakulla & WV & 0 \\
\hline 39 & Pelicaniformes & Ardeidae & Great egret & $\begin{array}{c}\text { Ardea alba } \\
\text { (Linnaeus,1758) }\end{array}$ & Thulo seto bakulla & $\mathrm{R}$ & $\mathrm{C}$ \\
\hline 40 & Pelicaniformes & Ardeidae & Cattle egret & $\begin{array}{c}\text { Bubulcus ibi } \\
\text { (Linnaeus, 1758) }\end{array}$ & Bastu bakulla & $\mathrm{R}$ & $\mathrm{C}$ \\
\hline 41 & Pelicaniformes & Ardeidae & Intermediate Egret & $\begin{array}{c}\text { Ardea intermedia } \\
\text { (Wagler, 1827) }\end{array}$ & Sano bakulla & $\mathrm{R}$ & $\mathrm{C}$ \\
\hline
\end{tabular}




\begin{tabular}{|c|c|c|c|c|c|c|c|}
\hline 42 & Pelicaniformes & Threskiornithidae & Black ibis & $\begin{array}{c}\text { Pseudibus papillosa } \\
\text { (Temminck,1824) }\end{array}$ & Karra sawari & WV & O \\
\hline 43 & Piciformes & Megalaimidae & $\begin{array}{c}\text { Blue-throated } \\
\text { Barbet }\end{array}$ & $\begin{array}{c}\text { Megalaima asiatica } \\
\text { (Latham, 1790) }\end{array}$ & Kuthukre & R \\
\hline 44 & Piciformes & Picidae & $\begin{array}{c}\text { Fulvous-brested } \\
\text { woodpecker }\end{array}$ & $\begin{array}{c}\text { Dendrocopos macei } \\
\text { (Vieillot, 1818) }\end{array}$ & Kastha kut & $\mathrm{R}$ \\
\hline 45 & Psittaciformes & Psittacidae & $\begin{array}{c}\text { Rose-ringed } \\
\text { Parakeet }\end{array}$ & $\begin{array}{c}\text { Psittacula krameri } \\
\text { (Scopoli, 1769) }\end{array}$ & Kanthe suga & R & C \\
\hline 46 & Strigiformes & Strigidae & Spotted Owlet & $\begin{array}{c}\text { Athene brama } \\
\text { (Temminck, 1821) }\end{array}$ & Laatkosero & R \\
\hline 47 & Strigiformes & Strigidae & Jungle Owlet & $\begin{array}{c}\text { Glaucidium radiatum } \\
\text { (Tickell, 1833) }\end{array}$ & Dundul & R \\
\hline 48 & Strigiformes & Strigidae & Brawn Hawk Owl & $\begin{array}{c}\text { Ninox scutulata } \\
\text { (Raffles, 1822) }\end{array}$ & Kaal pechak & R \\
\hline 49 & Suliformes & Phalacrocoracidae & Little Cormorant & $\begin{array}{c}\text { Phalacrocorax niger } \\
\text { (Vieillot, 1817) }\end{array}$ & Saano jalewa & WV \\
\hline
\end{tabular}

$\mathrm{SS}=$ Seasonal Status, $\mathrm{AB}=$ Abundance, $\mathrm{R}=$ Resident, $\mathrm{WV}=$ Winter Visitor, $\mathrm{SV}=$ Summer Visitor, $\mathrm{C}=$ Common, $\mathrm{FC}=$ Fairly Common, $\mathrm{O}=\mathrm{Occasional}, \mathrm{UC}=$ Uncommon, a = IUCN Red List status (2017) : a Near threatened, b vulnerable
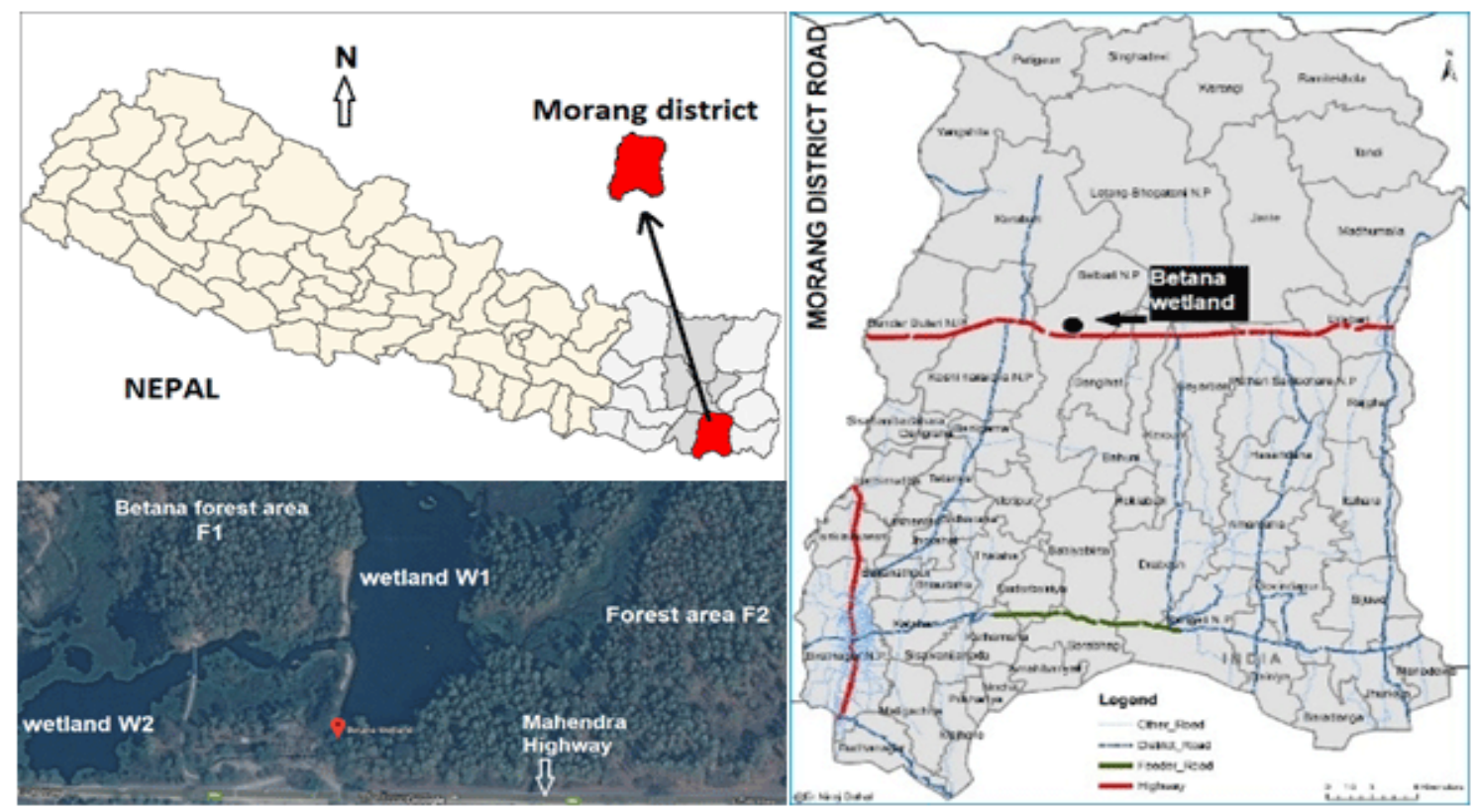

Figure 1: Map of study the site. 


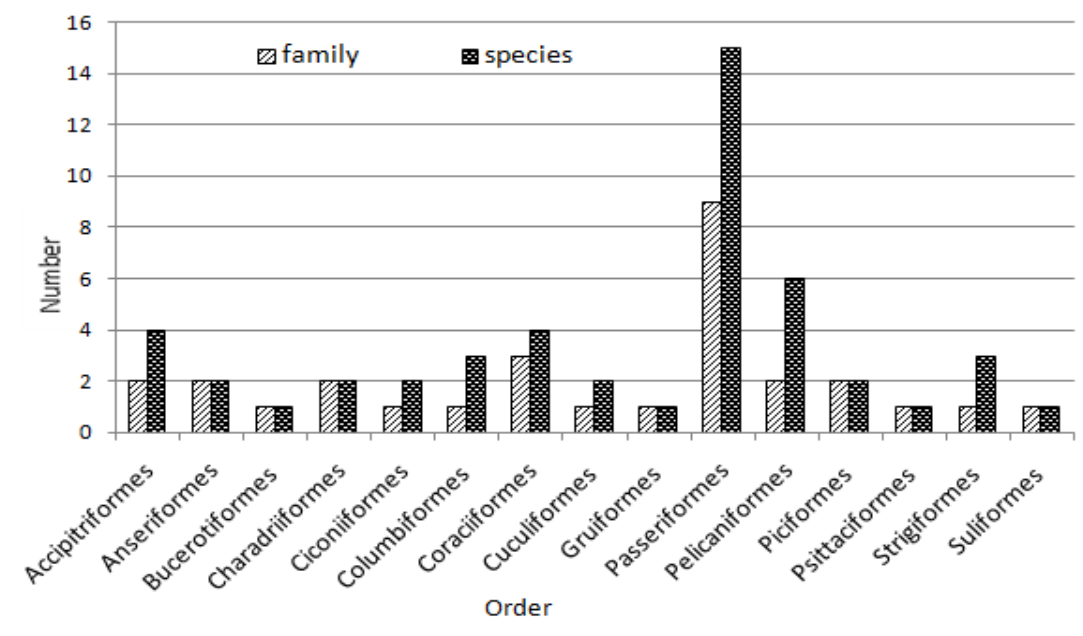

Figure 2: Avifaunal composition of Betana wetland (orderwise)

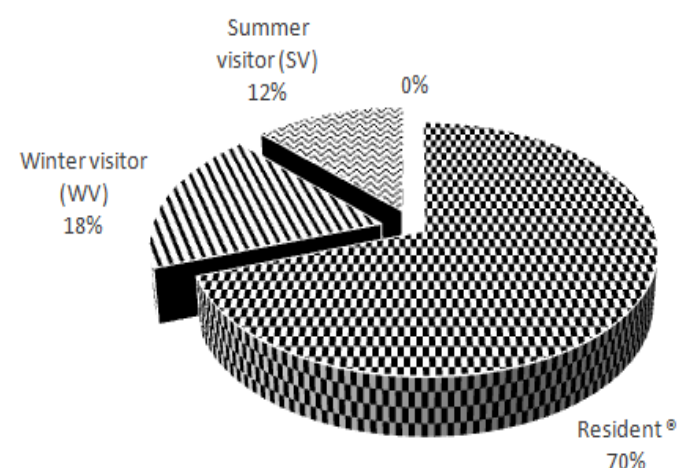

Figure 3: Pie showing seasonal status of avifauna on percentage basis

\section{Discussion}

In the present study most of the birds were found belong to the order Passeriformes including nine families and 15 species, followed by Coraciformes with three families and four species then Pelicaniformes with two families and six species. Of them, two species Grey-headed fish eagle (Icthyophaga humilis) and Lesser Adjutant Stork (Leptoptilos javanicus) are kept under near threatened (NT) and vulnerable (VU) categories of IUCN Red List status of threatened species respectively. In the previous study, 55 species of birds from 26 families and 10 orders were recorded but in the present study, 49 species of birds belonging to 30 families and 15 orders have been reported. Pokharel, et al. 2015 recorded Black Kite (Milvus migrans), Indian Roller (Coracius benghalensis) and White-throated Kingfisher (Halcyon smyrnensis) as very common species in the Betana wetland $[12,13]$. But this time these species were occasionally seen. This may be due to limitation of survey period or due to shortage of food resources (?). However, Red-vented Bulbul (Pycnonotus

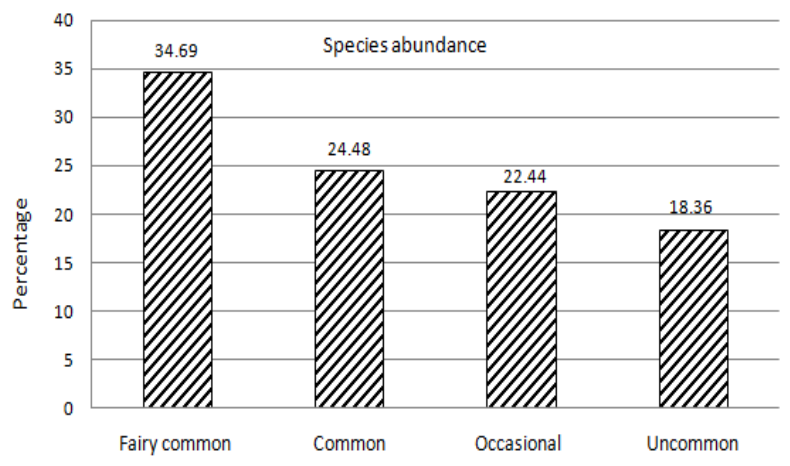

Figure 4: Species abundance of avifauna.

cafer), Rose-ringed Parakeet (Psittacula krameri), Black Drongo (Dicrurus macrocercus), Oriental Magpie Robin (Copsychuss aularis), Common Myna (Acridotherestristis), Great Egret (casmerodius albus), House Sparrow (Passer domesticus), Spotted Dove (Streptopelia chinensis) etc. were recorded as high population especially in winter season.

In the previous study, Out of 55 species, 44 species were resident, five species were winter visitor and six species were summer visitor but this time, out of 49 species, 34 species were resident $(70 \%)$, nine species $(18 \%)$ were winter visitor and six species $(12 \%)$ were summer visitor. Four more species of winter visitor birds were recorded this time.

\section{Conclusion}

Compared to the previous work, birds belonging to four more families and five more orders were recorded this time. However, total numbers of species were recorded less during the present study. The species richness was found higher in winter 
season than summer. This may be due to easy availability of food, suitable climate, temperature and migration of species. During the present survey, 49 species of birds belonging to 30 families and 15 orders have been recorded from the Betana wetland, which proves that the study area is one of the suitable habitats for avifaunal abundance. About $70 \%$ of total bird recorded was found to be the resident type and about $35 \%$ of total recorded bird species were found fairly common. The major threats to the avifauna in the study site were found to be deforestation, overgrazing, bird killing by using catapult and pollution due to recreational activities.

\section{Acknowledgements}

I am thankful to the local people who helped during field visits. I would like to acknowledge the Head, Department of Zoology, Central Campus of Technolgy, Dharan (TU) for providing the concerned literatures. Thanks are due to IDEA WILD ORG., USA for donating field gears.

\section{References}

1. Ali S, Ripley SD. Handbook of the Birds of India and Pakistan. 1986;5:1278. Oxford University Press.

2. Birdlife Nepal. Accessed $20^{\text {th }}$ Aug 2018.

3. Fleming RL(Sr.), Fleming RL (Jr.), Bangdel LS . Birds of Nepal with reference to Kashmir and Sikkim. First Adarsh Impression, Gaurav Offset, Delhi. 2000.
4. Grimmet R, Inskipp C, Inskipp T. Birds of Nepal. Helm Field Guide. Prakash Books, New Delhi. 2000.

5. Grimmet R, Inskipp C, Inskipp T, Baral HS .Birds of Nepal, Helm Field Guide. Revised edition, Christopher Helm, London. 2016.

6. Hutto RL, Pletscher SM, Hendricks P. A fixed-radius point count method for nonbreeding and breeding season use. Auk. 1986;103:593-602.

7. Hollis GE, Holland MM, Larson JS. Wise use of wetlands. J. Nature and Resource. 1998;24(1):2-13.

8. Pradhan P. A pocket photo guide to the birds of Nepal. Falcon Publication, Singapore. 2017:1-268.

9. Rai SK. Algal flora of Betana wetland, Morang, Nepal. J. Nepalese Journal of Bioscience. 2011;1:104-113.

10. Shrestha TK. Birds of Nepal. Field ecology. J. Natural History and Conservation. 2000;1:287.

11. Subba BR, Chhetri DC. Study of fauna of Betana village development committee, Morang, Nepal. A report submitted to APEC, Biratnagar. 2005.

12. Pokharel U. Diversity and conservation status of birds of Betana wetland area, Belbari, Morang, Nepal. M.Sc. Unpublished Thesis. Central Department of Zoology, Tribhuvan University, Kathmandu, Nepal. 2015.

13. Surana R, Subba BR, Limbu KP. Avian diversity during rehabilitation stage of Chimdi Lake, Sunsari, Nepal. J. Our Nature.2007; 5:75-80. 